and therefore I admit that if Mr. Bryan attaches his wheel and windlass to my pistin of constant mass, we should get

$$
\int \frac{\partial Q}{T}=0
$$

for each complete turn of the wheel. Whether that equation can be correctly said to express the second law, where there is only one independent variable, is a question of definition of the second law.

I said, also, that if we are at liberty to vary the mass of the pistin, we have two independent variables, but no longer a conservative system. Mr. Bryan, with greater generality, points out that the same effect would be produced by altering the gravitation-potential.

The objections to Clausius's proof generally cannot be more forcibly stated than they are in the Report. What is required is a definition of the time " $i$." The absence of that definition is to my mind not only an objection, but a quite fatal objection. If, as I proposed, we make

$$
i=\frac{v^{\frac{7}{3}}}{\mathrm{~T}^{\frac{1}{2}}}
$$

that answers the purpose for the very limited class of cases in which

$$
\partial \bar{\chi}=\frac{\overline{d \chi}}{d v} \partial v .
$$

A treatment of the subject in generalised coorodinates is as follows :-Let $y_{1} \ldots y_{1}$ be the unconstrainable, $q_{1} \ldots q_{r}$ the controllable coordinates, concerning which latter I assume, as does Boltzmann, that $\dot{q}$ or $\frac{d q}{d t}$, and also $\frac{d^{2} q}{d t^{2}}$ are to be neglected. Let $\chi$ be the potential, $\tau$ the kinetic energy, $T$ the mean value of $\tau$. Then we have generally

$$
\frac{\partial \mathrm{Q}}{\mathrm{T}}=\partial \log \mathrm{T}+\frac{\mathbf{I}}{\mathrm{T}}\left\{\partial \chi+\mathbf{\Sigma}\left(\frac{\overline{d t}}{d q}-\frac{\overline{d \chi}}{d q}\right) d q\right\} .
$$

In some cases the term $\frac{d \mathrm{~T}}{d q}$ does not appear, and therefore in this class of cases

$$
\frac{\partial \mathrm{Q}}{\mathrm{T}}=\partial \log \mathrm{T}+\frac{\mathrm{1}}{\mathrm{T}}\left(\partial \vec{\chi}-\Sigma \overline{d \chi} \frac{\bar{d}}{d q}\right) .
$$

Now let $f d y_{1} \ldots . d y_{n}$ be the chance that in the stationary motion with the $q$ 's constant, the coordinates shall lie between the limits $y_{1}$ and $y_{1}+d y_{1}$, \&c., so that

and

$$
\bar{\chi}=\iint \cdots \cdot x f d y_{1} \ldots . d y_{n}
$$

$$
\frac{\overline{d \chi}}{d q}=\iint \cdots \frac{d \chi}{d q} f d y_{1} \ldots \cdot d y_{n} .
$$

This makes

and

$$
\partial \bar{\chi}-\Sigma_{d \chi}^{d \bar{d}} \partial q=\iint \cdot \cdot \chi \partial \int d y_{1} \ldots . d y^{\prime} n,
$$

$$
\frac{\partial \mathrm{Q}}{\mathrm{T}}=\partial \log \mathrm{T}+\iint \ldots \frac{\chi}{\mathrm{T}} \partial f d y_{1} \ldots . d y_{n} .
$$

In order that $\frac{\partial Q}{T}$ may be a complete differential, we must make $t=\phi\left(\frac{\chi}{\mathrm{T}}\right)$, where $\phi$ is an arbitrary function. That is the general solution.

Now it will be found that this general solution agrees exactly with that given by Mr. Nichols. For his condition is

$$
\frac{\mathbf{I}}{\mathrm{T}}\left(\frac{d}{d v} \bar{\chi}-\frac{\overline{d \chi}}{d v}\right)=-\frac{d}{d \mathrm{~T}} \overline{\frac{d \chi}{d v}} \text {. }
$$

Now the use of these averages necessarily implies the existence of a function $f$, such that

$$
\bar{x}=\int f \chi d \sigma, \frac{\overline{d \chi}}{d v}=\int f \frac{d \chi}{d v} d \sigma,
$$

the integrations being with proper coordinates, and therefore

$$
\frac{d}{d v} \bar{x}-\frac{\overline{d \chi}}{d v}=\int \chi \frac{d f}{d v} d \sigma
$$

NO. I $26_{3}$, VOL. 49 ]
Mr. Nichols' condition may therefore, without loss of its generality, be put in the form

$$
\int \frac{\chi}{\mathrm{T}} \frac{d f}{d v} d \sigma=-\int \frac{d \chi}{d v} \frac{d f}{d \mathrm{~T}} d \sigma
$$

The general solution of this is

$$
f=\phi\left(\frac{\chi}{\bar{T}}\right)
$$

as before

That equation,

$$
f=\phi\left(\frac{\chi}{\mathrm{T}}\right),
$$

seems to me to define the limits of the second law for all cases in which the term $\frac{d \mathrm{~T}}{d q}$ does not appear in Lagrange's equations.

I hope if Mr. Bryan comes to discuss the virial proof, he will give his opinion on this point.

I think that the term $\frac{\mathrm{I}}{\mathrm{T}} \frac{d \tau}{d q}$, when it exists, can be made to lead to a somewhat similar condition. If, namely, $\mathrm{F}$ be the chance of a given combination of the velocities,

$$
\begin{aligned}
\frac{\mathrm{I}}{\mathrm{T}} \frac{\overline{d \tau}}{d q}=\int \frac{\mathrm{F}}{\mathrm{T}} \frac{d \tau}{d q} d \sigma & =-\int \frac{\tau}{\mathrm{T}} \frac{d \mathrm{~F}}{d q} d \sigma \\
& =-\int \frac{\tau}{\mathrm{T}} \partial \mathrm{F} d \sigma+\partial \log \mathrm{T},
\end{aligned}
$$

whence $F$ must be a function of $\frac{\tau}{T}$.

I have to thank Mr. Bryan for reminding me of Mr. Nichols' paper, which I had forgotten, though I had some discussion with its author at the time.

S. H. BURBURY.

\section{The Fauna of the Victoria Regia Tank in the Botanical Gardens.}

Prof. Lankester's account of the "Freshwater Medusa," in NATURE, December 7,1893 , shows how with very little trouble the interests of zoologists may be served by those who have the charge of botanical gardens like that in the Regent's Park. All that is necessary is to refrain from periodically cleaning out the tanks in which tropical water plants are grown. When these latter are imported from abroad they often carry with them various aquatic animals of novelty or interest like the medusa mentioned. This particular tank has recently produced quite a number of remarkable animals. Mr. Bousfield, some years since, found certain new or little known species of Dero therein, and more recently Prof. A. G. Bourne met with a new form of the Naid genus Pristina in the same tank. I have been able, thanks to the courtesy of Mr. Sowerby, to examine water and decaying weed therefrom on more than one occasion, and I discovered a series of rare or novel species of Oligochata. The most remarkable form was one which I described a year or two since as a new genus Branchiura; this worm, with the general characters of a Tubifex, possesses a row of dorsal and ventral branchial processes, besides showing other points of interest. In the same sample of water were large quantities of a Naid, called by its original describer, Prof. Bourne, who met with it in the town of Calcutta, Chetobranchus semperi. This worm has also a series of branchiæ, but they are lateral in position, and enclose the long dorsal setæ of the Annelid, thus suggesting the parapodia of the marine Chrtopods. I have also found the rare species Asolosoma niveum in the same locality, and a freshwater Nemertine ( $\%$ Tetrasteruma aquarum dulcium), besides a number of Oligochæta which I did not at the time identify.

FRANK E. BEIDARD.

\section{Rudimentary (Vestigial) Organs.}

PROF. HARTOG'S letter in your issue of the 28 th ult. is interesting as an illustration of the extreme danger of regarding an organ as vestigial and functionless merely because our superficial investigations have not revealed to us its use.

If Prof. Hartog had examined the distal knob of the modern eyeglass, even by the old-fashioned macroscopic methods, he would have seen that there passes through a screw, whose function is to hold the two sides of the oval frame together, and thus retain the lens in place.

The knob is also useful as a convenient point to lay hold of 